\title{
Slaughter value and meat quality of Muscovy ducks, Pekin ducks and their crossbreeds, and evaluation of the heterosis effect
}

\begin{abstract}
The studies were conducted on 200 Muscovy ducks, 100 Pekin ducks of the A-44 strain and 200 their crossbreeds (sex ratio: 1:1). The ducks were fed complete commercial diets, appropriate for respective species. Slaughter and slaughter value estimation were performed at the age of: 7 weeks - for males and females of the A-44 strain, 10 weeks - for Muscovy females, and 12 weeks - for Muscovy males and crossbreeds ( $\hat{\partial}$ and $\phi$ ). Analysis of the chemical composition, physicochemical properties and sensory properties of meat was made on breast muscles. The heterosis effect on the slaughter value and meat quality was additionally estimated in hybrids.

The highest live body weights were reported for Mucovy drakes (4450 g), and the lowest - for ducks from this genetic group (ca. $2400 \mathrm{~g}$ ). The crossbreeds were characterized by average body weights (2980 g), but the highest relative meatiness (ca. 54\%) and the lowest fatness (са. 20\%) of carcasses. The best sensory properties were reported for muscles of ducks of the A-44 strain. A favorable heterosis effect was observed in crossbreeds: positive in relation to meatiness and negative in relation to fatness, undesirable in the customers' opinion. No favorable heterosis effect was noted in most meat quality traits.
\end{abstract}

Key Words: Muscovy ducks, Pekin ducks, crossbreeds, slaughter value, meat quality, heterosis

\section{Zusammenfassung}

Titel der Arbeit: Schlachtwert und Fleischqualität von Bisamenten, Pekingenten und ihren Kreuzungen und Schätzung von Heterosiseffekten

In die Untersuchungen zum Schlachtwert wurden Bisamenten $(n=200)$, Pekingenten Linie A-44 $(n=100)$ und Kreuzungstiere beider Populationen $(n=200)$ bei einem Geschlechtsverhältnis von 1:1 einbezogen. Die Enten erhielten artgerechtes Mischfutter. Die Schlachtung und Schlachtkörperbeurteilung erfolgte populationsabhängig zu unterschiedlichen Zeitpunkten: Tiere der Linie A-44 im Alter von 7 Wochen, Bisamhennen im Alter von 10 Wochen während Bisamhähne und Kreuzungstiere mit 12 Wochen geschlachtet wurden. Die chemischen Fleischanalysen, die physisch-chemische und sensorische Merkmalsbeurteilung erfolgte am Brustfleisch. Für Merkmale des Schlachtwertes und der Fleischqualität der Kreuzungstiere wurden Heterosiseffekte geschätzt. Vor der Schlachtung erreichten Bisamerpel das höchste (4450 g) und Bisamhennen das niedrigste (2400 g) Körpergewicht. Kreuzungsenten erreichten ein Durchschnittsgewicht von 2980 g, aber die relativ beste Fleischigkeit (ca. $54 \%$ ) und geringste Verfettung (va. $20 \%$ ). Die besten sensorischen Eigenschaften besaßen die Muskeln der Linie A-44. Bei den Entenkreuzungen ergaben sich Heterosiseffekte in Bezug auf höhere Werte für die Muskulatur und niedrigere für den Fettgehalt. Keine Heterosiseffekte konnten für die Fleischqualitätsmerkmale nachgewiesen werden.

Schlüsselwörter: Bisamenten, Pekingenten, Kreuzung, Schlachtwert, Fleischqualität, Heterosis

\section{Introduction}

Duck meat production is based mainly on commercial crossbreeds of different Pekin strains (KSIĄŻKIEWICZ, 1995; PINGEL, 1997; ZEIDLER, 1998). The most ducks are reared in Asia and Europe, especially France. As regards Pekin duck production in Poland, a very important role is played by ducks of the A-44 strain, imported from the United Kingdom (KSIĄŻKIEWICZ, 1995). 
In Europe, Pekin ducks are more and more often substituted for Muscovy ducks originating from America. The popularity of Muscovy ducks increased considerably when the French company Grimaud Fre'res, in cooperation with INRA, started (1968) a project aimed at improving this species (BOISDRON and GRIMAUD, 1992; HOFFMAN, 1995).

A growing interest in crossbreeds of Muscovy drakes with Pekin ducks could be observed in the last years of the previous century (BABILE et al., 1988; BAEZA et al., 2000; MAZANOWSKI and BERNACKI, 2000; WAWRO et al., 2001). Such hybrids, called Mullards, were used in the past mainly for fattened liver production, nowadays they are also used for broiler production (BABILE et al., 1988; BAEZA et al., 2000; PINGEL and TRETNER, 1987; WAWRO et al., 2001; WILKIEWICZ-WAWRO, 1994). For example, of all duck meat produced in France in 1997, 35\% was meat from Mullards (BAEZA et al., 2000). The production of these hybrids becomes more and more popular also in other countries of Western and Central Europe.

The crossbreeding of Muscovy drakes with Pekin ducks is aimed at evoking heterosis mainly in slaughter value traits. WAWRO et al. (2000) observed favorable positive heterosis in hybrids in relation to the blood content of hemoglobin and total lipids, and the activity of aminotransferases in the blood. Negative heterosis noted in relation to the cholesterol content and the activity of phosphatases in the blood serum of Mullards seems to be desirable as well.

The aim of the present study was to compare the slaughter value of Muscovy ducks, Pekin ducks of the A-44 strain and their crossbreeds, as well as to evaluate the heterosis effect in these crossbreeds with respect to slaughter value traits.

\section{Material and Methods}

The experimental material were 200 (100 0 and 100 o) Muscovy ducklings, 100 (50 $\delta$ and 50 \&) A-44 strain ducklings and 200 crossbreeds (100 $\delta$ and 100 \&) of Muscovy drakes with A-44 ducks. One-day-old, sexed ducklings (sex ratio: 1:1) were weighed and marked. They were reared in a house, in rearing pens (males and females separately), on rye straw litter, under appropriate light, temperature and air humidity conditions. They had free access to water. The A-44 strain ducks and Muscovy ducks to the age of three weeks were fed a complete diet " $A$ " containing $19.70 \%$ crude protein and $12.40 \mathrm{MJ}$ metabolizable energy. Starting from the $4^{\text {th }}$ week, the Muscovy ducks were fed a pelleted diet "B" (pellet diameter $3.5 \mathrm{~mm}$ ) containing $16.10 \%$ crude protein and 12.23 MJ metabolizable energy, whereas the A-44 strain ducks - a friable complete diet " $\mathrm{B}$ ". The crossbreeds were fed a diet " $\mathrm{C}$ ' to the age of six weeks and a diet " $\mathrm{D}$ " from the $7^{\text {th }}$ week. The diets contained 18.50 and $17.80 \%$ crude protein and 12.02 and 11.59 MJ metabolizable energy, respectively. The experimental diets (A,B,C and D) contained all vitamins and minerals indispensable for proper growth and development of ducks.

The males and females of the A-44 strain were reared to the age of seven weeks, the Muscovy females - to ten weeks, the Muscovy males and hybrids $(\hat{o} \mathrm{i}$ + ) - to 12 weeks. When the rearing period was over, $20 \hat{\gamma}$ and $20 \%$ were selected from each genetic group for slaughter and slaughter value estimation, by layer sampling (preceded by arranging the ducks into an ascending statistical sequence by body weights), which ensured sample randomness and representativeness. Fasted (ca. 12 h) 
ducks were weighed, slaughtered, plucked and eviscerated. The weights of carcass, giblets and abdominal fat were determined (Table 1).

Table 1

Experimental design (Versuchsdatensatz)

\begin{tabular}{|c|c|c|c|c|c|c|}
\hline \multirow{3}{*}{ Specification } & \multicolumn{6}{|c|}{ Ducks and sex } \\
\hline & \multicolumn{2}{|c|}{ Muscovy } & \multicolumn{2}{|c|}{ A-44 strain } & \multicolumn{2}{|c|}{ hybrids } \\
\hline & $\hat{0}$ & $q$ & $\hat{0}$ & o & $\hat{0}$ & 9 \\
\hline Number of birds: & & & & & & \\
\hline Reared & 100 & 100 & 50 & 50 & 100 & 100 \\
\hline Slaughtered and dissected & 20 & 20 & 20 & 20 & 20 & 20 \\
\hline Age at slaughter (weeks) & 12 & 10 & 7 & 7 & 12 & 12 \\
\hline
\end{tabular}

The carcasses were chilled at a temperature of $4^{0} \mathrm{C}$ for 18 hours, then weighed again and divided into parts according to the methodology described by LEWCZUK et al. (1992). Detailed dissection into meat, skin with subcutaneous fat, intermuscular fat and bones was performed on the neck, breast, right leg, right wing, and back. The weight of particular tissue components in legs and wings was calculated multiplying by two the results obtained for the right parts. The calculations concerning the edible parts included muscles, skin with subcutaneous and intermuscular fat, and giblets (liver, gizzard and hart).

The carcass dressing percentage I and II was calculated according to the following formulas: I - ratio of the weight of an eviscerated carcass with the neck and giblets to the live body weight; II - ratio of the weight of a chilled carcass without the neck to the live body weight.

The basic chemical composition of breast muscles ( $m$. pectoralis major and minor); dry matter, fat, crude protein and ash were determined by conventional methods. The physicochemical properties of muscles were determined in the following way: water holding capacity (WHC) - by the GRAU and HAMM method modified by POHJA and NINIVAARA (1957); color brightness - with a "Specol" spectrometer with a remission attachment $\mathrm{R} 45 / 0$, at a wavelength of $560 \mathrm{~nm}$; and $\mathrm{pH}$ - with a Radiometer pH-meter with PHC 4406 electrodes. The sensory properties of muscles were evaluated on a five-point scale, according to BARYŁKO-PIKIELNA et al. (1964).

Statistical analyses (Statsoft, 1995, Statistica, ver. 6,0) included:

a) calculation of the arithmetic means $(\bar{x})$ and standard deviations (s) of the traits examined;

b) determination of the significance of differences between means for the traits examined in males and females in particular genetic groups and between these groups ( $\widehat{\partial}$ and + ) ; analysis of variance (ANOVA) ion a one-factor design, with two or three levels, respectively, according to the linear model $Y_{i j}=\mu+A_{j}+e_{i j}$, wheer: $Y_{i j}$ - phenotypic value of trait; $\mu$-total mean; $A_{j}$ - effect of jth sex or group of ducks; $\mathrm{e}_{\mathrm{ij}}$ - random observation error.

The heterosis effect in crossbreeds was determined by the following formula (WAWRO et al., 2000):

$$
\mathrm{He}=\frac{200 \mathrm{Pm}}{\mathrm{A}+\mathrm{B}}-100, \text { where }
$$

He - heterosis effect (\%) ; Pm - mean value of a trait in crossbreeds; A, B- mean value of trait in paternal and maternal strain ducks. To calculate the heterosis effect in males, the value of a trait of Mullard drakes was taken as Pm, the value of a trait of 
Muscovy drakes - as A, and the value of a trait of A-44 drakes - as B. In females the calculations were performed similarly: Pm - $\rightarrow$ hybrids; A - $q$ Muscovy; B - $\rightarrow$ A-44. The total He for both sexes was determined using the relevant data for both males and females.

\section{Results}

Among the duck groups examined, the highest body weights before slaughter were reported for the Muscovy drakes (4450 g, Table 2), and the lowest - for the Muscovy ducks (ca. 2400 g). As mentioned in Material and Methods, the ducks of the A-44 strain, with average body weights of $2870 \mathrm{~g}$, were slaughtered at seven weeks of age, which is consistent with the current trends to shorten the rearing period of Pekin ducks. Similarly as in the Muscovy ducks, the males of the A-44 strain were heavier than the females. However, the sexual dimorphism concerning this trait turned out to be considerably lower in the A-44 strain (ca. $300 \mathrm{~g}$ ) than in the Muscovy ducks (ca. 2050g). The crossbreeds were characterized by higher body weights ( $\bar{x}$ ca. $2980 \mathrm{~g}$ ) than the A-44 strain ducks. The sexual dimorphism observed in body weights between crossbred males and females was similar as in the A-44 strain ducks.

\section{Table 2}

Body weights of ducks before slaughter and weight of carcass, giblets, abdominal fat and edible parts, and carcass dressing percentage (Körper- und Schlachtkörpergewicht, Talgfett, Innereien und essbare Teile sowie Schlachtleistung der Enten)

\begin{tabular}{|c|c|c|c|c|c|c|c|c|c|c|}
\hline \multirow{3}{*}{ Specification } & \multirow{3}{*}{$\begin{array}{c}\text { Statis- } \\
\text { tics }\end{array}$} & \multicolumn{9}{|c|}{ Ducks and sex } \\
\hline & & \multicolumn{3}{|c|}{ Muscovy } & \multicolumn{3}{|c|}{ A-44 strain } & \multicolumn{3}{|c|}{ hybrids } \\
\hline & & $\sigma^{1}$ & 9 & ơ & $\hat{0}$ & 9 & jo & o & 9 & 万o+ \\
\hline $\begin{array}{l}\text { Weight of (g): } \\
\text { - body }\end{array}$ & $\begin{array}{l}\bar{X} \\
s\end{array}$ & $\begin{array}{c}4451^{* *} \\
355.6\end{array}$ & $\begin{array}{l}2397 \\
112.4\end{array}$ & $\begin{array}{l}3424^{\mathrm{A}} \\
1022.1\end{array}$ & $\begin{array}{c}3008^{* *} \\
231.0\end{array}$ & $\begin{array}{l}2726 \\
200.6\end{array}$ & $\begin{array}{l}2868 \\
253.8\end{array}$ & $\begin{array}{c}3150 * * \\
370.4\end{array}$ & $\begin{array}{l}2816 \\
343.3\end{array}$ & $\begin{array}{l}2983^{\text {B }} \\
391.1\end{array}$ \\
\hline - carcass & $\begin{array}{c}\bar{X} \\
\mathrm{~s}\end{array}$ & $\begin{array}{c}3158^{* *} \\
276.6\end{array}$ & $\begin{array}{l}1698 \\
87.8\end{array}$ & $\begin{array}{l}2428^{\mathrm{A}} \\
766.8\end{array}$ & $\begin{array}{c}2059 * * \\
149.5\end{array}$ & $\begin{array}{l}1879 \\
149.1\end{array}$ & $\begin{array}{c}1969^{\mathrm{Bb}} \\
170.9\end{array}$ & $\begin{array}{c}2136 * * \\
273.8\end{array}$ & $\begin{array}{r}1966 \\
256.4\end{array}$ & $\begin{array}{c}2051^{\text {Ba }} \\
275.5\end{array}$ \\
\hline - giblets & $\begin{array}{l}\bar{X} \\
s\end{array}$ & $\begin{array}{c}199.3^{* *} \\
22.6\end{array}$ & $\begin{array}{c}108.1 \\
11.3\end{array}$ & $\begin{array}{c}153.7 \\
49.4\end{array}$ & $\begin{array}{c}168.2^{* *} \\
20.8\end{array}$ & $\begin{array}{c}151.8 \\
16.7\end{array}$ & $\begin{array}{c}160.0 \\
20.1\end{array}$ & $\begin{array}{c}181.1^{* *} \\
28.1\end{array}$ & $\begin{array}{c}162.6 \\
19.6\end{array}$ & $\begin{array}{c}171.8 \\
25.7\end{array}$ \\
\hline - abdominal fat & $\begin{array}{l}\bar{X} \\
S\end{array}$ & $\begin{array}{c}84.5^{* * *} \\
33.7\end{array}$ & $\begin{array}{l}45.8 \\
12.0\end{array}$ & $\begin{array}{c}65.1^{\mathrm{A}} \\
31.7^{-}\end{array}$ & $\begin{array}{c}34.5 \\
9.8\end{array}$ & $\begin{array}{c}37.5 \\
6.7\end{array}$ & $\begin{array}{c}36.0^{\mathrm{B}} \\
10.0\end{array}$ & $\begin{array}{l}4.5 \\
3.8\end{array}$ & $\begin{array}{l}7.9 \\
6.7\end{array}$ & $\begin{array}{c}6.2^{\mathrm{C}} \\
5.3\end{array}$ \\
\hline - edible parts & $\begin{array}{l}\bar{X} \\
s\end{array}$ & $\begin{array}{c}2676^{* *} \\
249.1\end{array}$ & $\begin{array}{l}1456 \\
86.5\end{array}$ & $\begin{array}{l}2066^{\mathrm{A}} \\
645.2\end{array}$ & $\begin{array}{l}1701 * \\
128.8\end{array}$ & $\begin{array}{l}1600 \\
111.4\end{array}$ & $\begin{array}{l}1651^{\mathrm{C}} \\
135.9\end{array}$ & $\begin{array}{l}1781 * \\
252.2\end{array}$ & $\begin{array}{r}1624 \\
231.7\end{array}$ & $\begin{array}{l}1703^{\mathrm{B}} \\
251.9\end{array}$ \\
\hline $\begin{array}{l}\text { Carcass dressing } \\
\text { percentage }(\%) \text { : }\end{array}$ & & & & & & & & & & \\
\hline$x$ & $\begin{array}{l}\bar{X} \\
s\end{array}$ & $\begin{array}{l}74.2 \\
1.25\end{array}$ & $\begin{array}{l}74.1 \\
1.48\end{array}$ & $\begin{array}{l}74.1 \\
1.35\end{array}$ & $\begin{array}{l}73.5 \\
1.43\end{array}$ & $\begin{array}{l}74.1 \\
0.99\end{array}$ & $\begin{array}{l}73.8 \\
1.23\end{array}$ & $\begin{array}{c}73.9 * * \\
2.09\end{array}$ & $\begin{array}{l}76.1 \\
0.81\end{array}$ & $\begin{array}{l}75.0 \\
1.93\end{array}$ \\
\hline II & $\begin{array}{l}\bar{X} \\
S\end{array}$ & $\begin{array}{c}58.6^{* *} \\
2.20\end{array}$ & $\begin{array}{l}60.9 \\
1.22\end{array}$ & $\begin{array}{c}59.7^{\mathrm{A}} \\
2.10\end{array}$ & $\begin{array}{l}59.8 \\
1.64\end{array}$ & $\begin{array}{l}60.3 \\
1.57\end{array}$ & $\begin{array}{c}60.1^{\mathrm{A}} \\
1.59\end{array}$ & $\begin{array}{c}56.1^{* *} \\
1.76\end{array}$ & $\begin{array}{l}58.5 \\
1.26\end{array}$ & $\begin{array}{c}57.3^{* *} \\
1.94\end{array}$ \\
\hline
\end{tabular}

Means followed by different letters ( genetic groups) or * (sex) are significantly different:

capital letters or $* *$ - at $\alpha=0.01$; small letters or * at $\alpha=0.05$

Differences in the body weights of the experimental ducks affected the other traits analysed (Table 2). The heaviest carcasses and the highest edible part content $(\overline{\mathrm{x}}=$ $2676 \mathrm{~g}$ ) of a carcass were reported for the Muscovy drakes, whereas the lightest carcasses and the lowest edible part content $(\overline{\mathrm{x}}=1456 \mathrm{~g})$ of a carcass - for the Muscove ducks. The Muscovy ducks were also characterized by significantly higher 
weight of abdominal fat (total mean for $\delta$ and $q$ ca. $65 \mathrm{~g}$ ), than the A-44 strain ducks (36 g) and Mullards (6 g). Carcass dressing percentage I was at a similar level (ca. 74 $\%)$ in all groups.

The highest weight of breast muscles (543 g), leg muscles (455 g), and meat (1555 g) was found in carcasses of the Muscovy males (Table 3). Carcasses of the male and female crossbreeds contained significantly more meat (by 277 g), breast muscles (by over $100 \mathrm{~g}$ ) and leg muscles (by $34 \mathrm{~g}$ ), compared with the A-44 ducks.

Carcass fatness showed different tendencies than their meatiness (Table 2). The total weight of subcutaneous fat with skin in carcasses of both males and females turned out to be significantly lower in the crossbreeds ( $\bar{x}$ ca. $400 \mathrm{~g}$ ) than in the Muscovy and A44 ducks ( $\overline{\mathrm{x}}>600 \mathrm{~g})$. In carcasses of the Mullards, the meat to skin with fat ratio was 2.7 (Table 3). In the Muscovy ducks this ratio was 1.8, and in the A-44 strain ducks 1.4 only. This train was also characterized by the least favorable meat to bones ratio $(2.5: 1)$. On the other hand, the advantage of the Muscovy ducks is a very good edible parts to bones ratio $(\overline{\mathrm{x}}>6.0)$.

Table 3

Carcass tissue composition (Schlachtkörperzusammensetzung)

\begin{tabular}{|c|c|c|c|c|c|c|c|c|c|c|}
\hline \multirow{3}{*}{ Specification } & \multirow{3}{*}{$\begin{array}{l}\text { Sta- } \\
\text { tis- } \\
\text { tics }\end{array}$} & \multicolumn{9}{|c|}{ Ducks and sex } \\
\hline & & \multicolumn{3}{|c|}{ Muscovy } & \multicolumn{3}{|c|}{ A-44 strain } & \multicolumn{3}{|c|}{ hybrids } \\
\hline & & $\hat{0}$ & 9 & jo+ & $\hat{0}$ & 9 & 1우 & $\hat{0}$ & 웅 & jo+ \\
\hline \multirow{3}{*}{$\begin{array}{l}\text { Weight in carcass of (g): } \\
\text { - breast muscles }\end{array}$} & & ** & & Aa & & & B & & & $\mathbf{A b}$ \\
\hline & $\overline{\mathrm{X}}$ & 542.7 & 272.5 & 407.6 & 275.5 & 256.3 & 265.9 & 368.0 & 367.7 & 367.8 \\
\hline & s & 54.87 & 30.85 & 143.76 & 33.06 & 28.14 & 31.43 & 103.64 & 109.43 & 105.08 \\
\hline \multirow[t]{2}{*}{ - leg muscles } & $\overline{\mathrm{X}}$ & $454.8^{* *}$ & 255.3 & $355.0^{\mathrm{A}}$ & $247.817^{*}$ & 233.5 & $240.6^{C}$ & $283.3^{*}$ & 266.7 & $275.0^{\mathrm{B}}$ \\
\hline & $\mathrm{s}$ & 43.25 & 23.46 & 106.68 & .54 & 21.97 & 20.67 & 44.48 & 38.03 & 41.72 \\
\hline \multirow[b]{2}{*}{ - total meat } & $\overline{\mathrm{X}}$ & $1554.8^{* *}$ & 824.4 & $1189.6^{\mathrm{A}}$ & $865.2^{* *}$ & 815.0 & $840.1^{\text {B }}$ & $1179.8^{*}$ & 1054.7 & $1117.3^{\mathrm{A}}$ \\
\hline & $\begin{array}{l}A \\
\mathrm{~s}\end{array}$ & 123.54 & 63.64 & 382.58 & 68.87 & 54.36 & 65.44 & 164.82 & 148.71 & 167.37 \\
\hline \multirow{2}{*}{ - bones } & $\overline{\mathrm{X}}$ & $439.6^{* *}$ & 227.8 & 333.7 & $369.0^{* *}$ & 302.2 & 335.6 & 355.8 & 343.9 & 349.9 \\
\hline & $\mathrm{s}$ & 34.68 & 24.47 & 111.26 & 39.67 & 34.66 & 49.33 & 43.44 & 37.07 & 40.34 \\
\hline \multirow[t]{2}{*}{ - skin with fat } & $\overline{\mathrm{X}}$ & $810.9^{* *}$ & 459.5 & $635.2^{\mathrm{A}}$ & 621.7 & 582.2 & $602.0^{\mathrm{A}}$ & 409.2 & 393.0 & $401.1^{\mathrm{B}}$ \\
\hline & $\mathrm{s}$ & 117.24 & 56.29 & 199.90 & 60.43 & 64.45 & 63.99 & 80.04 & 84.22 & 81.50 \\
\hline \multirow{2}{*}{ - intermuscular fat } & & $269^{*}$ & 177 & $\begin{array}{r}\mathrm{A} \\
22\end{array}$ & 112 & 159 & $13 \stackrel{B}{6}$ & & & $\begin{array}{r}\mathrm{C} \\
64\end{array}$ \\
\hline & $\begin{array}{l}X \\
S\end{array}$ & $\begin{array}{l}20.9 \\
8.06\end{array}$ & 9.67 & $\begin{array}{l}22.0 \\
9.95\end{array}$ & $\begin{array}{l}11.2 \\
8.21\end{array}$ & $\begin{array}{l}10.9 \\
10.84\end{array}$ & $\begin{array}{l}13.6 \\
9.66\end{array}$ & $\begin{array}{c}6.6 \\
41.96\end{array}$ & $\begin{array}{c}6.2 \\
3.96\end{array}$ & $\begin{array}{c}6.4 \\
4.49\end{array}$ \\
\hline Ratios: & & * & & B & & & C & * & & \\
\hline \multirow{2}{*}{ - meat to skin with fat } & $\overline{\mathrm{X}}$ & 1.9 & 1.7 & 1.8 & 1.4 & 1.4 & 1.4 & 2.8 & 2.6 & 2.7 \\
\hline & s & 0.26 & 0.29 & 0.28 & 0.14 & 0.13 & 0.13 & 0.31 & 0.35 & 0.34 \\
\hline \multirow[t]{2}{*}{ - meat to bones } & $\overline{\mathrm{X}}$ & $3.5^{*}$ & 3.6 & $\begin{array}{r}\text { A } \\
3.6\end{array}$ & $2.3^{* *}$ & 2.7 & $\begin{array}{r}\text { C } \\
2.5\end{array}$ & 3.3 & 3.1 & $\begin{array}{r}\text { B } \\
3.2\end{array}$ \\
\hline & $\mathrm{s}$ & 0.24 & 0.49 & 0.39 & 0.23 & 0.22 & 0.28 & 0.47 & 0.40 & 0.45 \\
\hline \multirow{3}{*}{ - edible parts to bones } & & $*$ & & A & * & & B & $*$ & & B \\
\hline & $\overline{\mathrm{X}}$ & 6.1 & 6.4 & 6.2 & 4.6 & 5.3 & 4.9 & 5.0 & 4.7 & 4.9 \\
\hline & $\mathrm{s}$ & 0,34 & 0.79 & 0.63 & 0.44 & 0.50 & 0.57 & 0.63 & 0.63 & 0.64 \\
\hline
\end{tabular}

Means followed by different letters ( genetics groups) or * (sex) are significantly different:

capital letters or $* *$ - at $\alpha=0.01$; small letters or * - at $\alpha=0.05$

An analysis of the relative percentage of the components (expressed as a carcass weight percentage) shows that carcasses of the crossbreeds were characterized by the highest content of breast muscles ( $\bar{x}$ ca. 18\%) and total meat ( $\bar{x}$ ca. $54 \%$ ) (Fig.). The breast muscle and total meat content of carcasses was at a slightly lower level in 
carcasses of the Muscovy ducks ( $\bar{x}$ ca. $17 \%$ and ca. $49 \%$ respectively) and at a much lower level in those of the A-44 strain ones ( $\bar{x} 13.5 \%$ and $43.5 \%$ respectively).
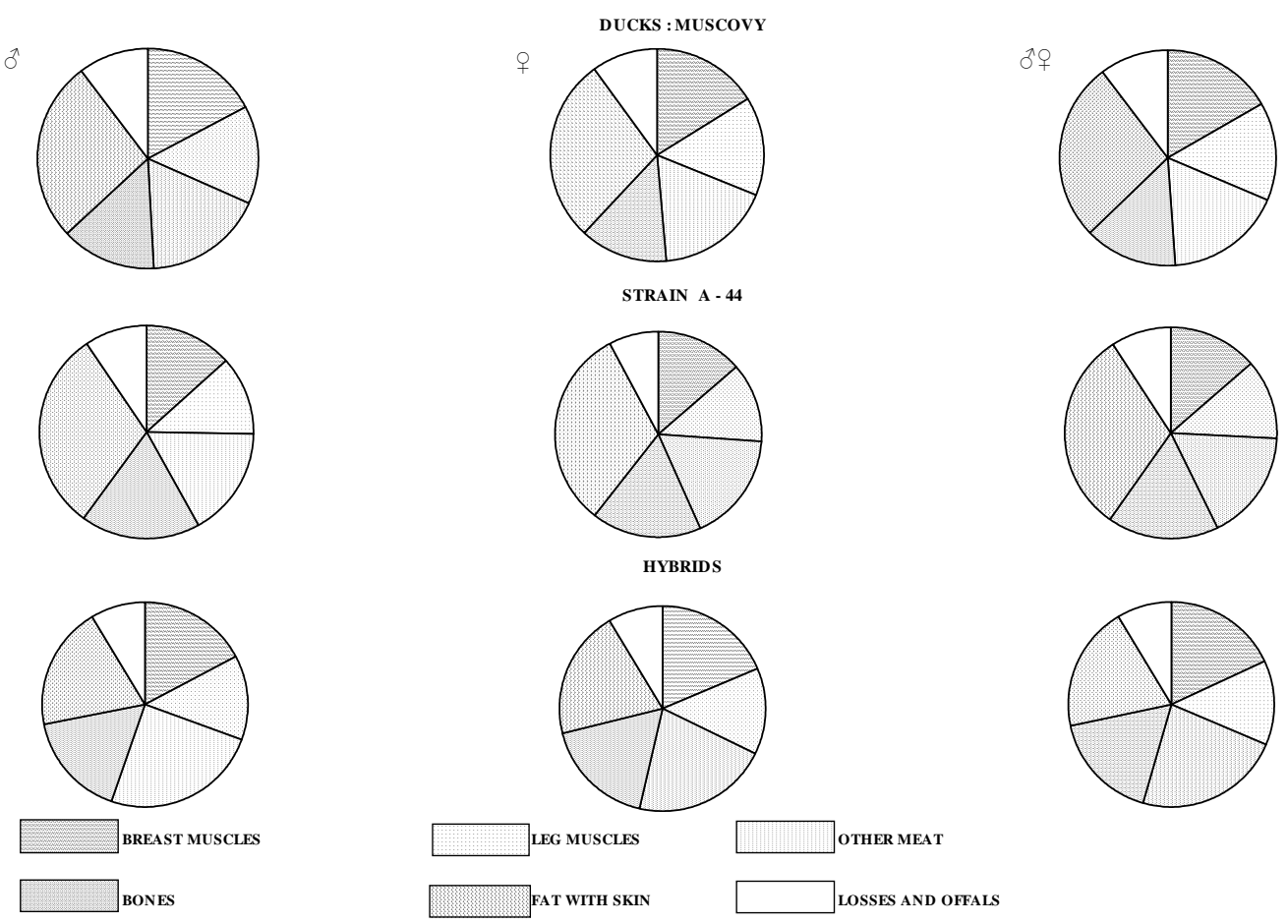

Fig.: Percentage of meat, fat with skin, and bones in carcasses (carcass weight $=100 \%$ ) (Anteile des Fleisches, Fettes mit Haut und der Knochen im Schlachtkörper ( Schlachtkörper Masse = 100\%))

The percentage of fat with skin displayed a different tendency than the meat content of duck carcasses. It was the highest ( $\bar{x}>30 \%$ ) in the A-44 strain ducks, and the lowest ( $\bar{x}$ ca. $20 \%$ ) - in the crossbreeds (Fig.). A certain defect of the Mullards seems to be a high percentage of bones in their carcasses.

The crude protein content was the highest $(\bar{x}=19.5 \%)$ in breast muscles of the Muscovy ducks and Mullards, and significantly lower $(\bar{x}=19.0 \%)$ - in breast muscles of the Pekin ducks $(\bar{x}=19.0 \%)$ (Table 4$)$. The meat from the crossbreeds was also characterized by a significantly higher dry matter content, compared with the meat from the Muscovy and Pekin ducks. The levels of fat and ash in the muscles were similar in the males and females, and within particular genetic groups.

The physicochemical properties of the duck muscles were different (Table 4). The meat from the Pekin ducks was characterized by better water holding capacity, i.e. lower drip losses $\left(2.3 \mathrm{~cm}^{2}\right)$ than the meat from the Muscovy ducks and Mullards $(\overline{\mathrm{x}}>$ $\left.2.6 \mathrm{~cm}^{2}\right)$.

The $\mathrm{pH}$ values of muscles (Table 4) were relatively low $(\overline{\mathrm{x}}<5.8)$, which is good taking into account the meat shelf life. The meat from the Muscovy ducks showed the lowest $\mathrm{pH}(\overline{\mathrm{x}}=5.7)$. Compared with the A-44 strain ducks and Mullards, their meat was also characterized by the highest color differences.

A sensory evaluation of breast muscles shows that the Pekin ducks demonstrated a tendency towards better meat quality (Table 5). Muscles of the A-44 ducks were characterized by not only higher tenderness and juiciness, but also better palatability than those of the Muscovy ducks and Mullards. 
Table 4

Chemical composition and physicochemical properties of duck muscles (Analyse und physisch-chemische Eigenschaften der Entenmuskel)

\begin{tabular}{|c|c|c|c|c|c|c|c|c|c|c|}
\hline \multirow{3}{*}{ Specification } & \multirow{3}{*}{$\begin{array}{l}\text { Statis- } \\
\text { tics }\end{array}$} & \multicolumn{9}{|c|}{ Ducks and sex } \\
\hline & & \multicolumn{3}{|c|}{ Muscovy } & \multicolumn{3}{|c|}{ A-44 strain } & \multicolumn{3}{|c|}{ hybrids } \\
\hline & & $\hat{0}$ & 우 & jot & $\hat{0}$ & ㅇ & 수오 & $\hat{0}$ & 9 & jo오․ \\
\hline \multirow{3}{*}{ Crude protein (\%) } & & & & $a^{a}$ & & & & & & a \\
\hline & $\overline{\mathrm{X}}$ & 19.6 & 19.3 & 19.5 & 18.9 & 19.1 & 19.0 & 19.5 & 19.5 & 19.5 \\
\hline & $\mathrm{S}$ & 0.66 & 0.41 & 0.55 & 0.77 & 0.63 & 0.69 & 0.78 & 0.47 & 0.70 \\
\hline \multirow{2}{*}{ Fat } & $\overline{\mathrm{X}}$ & 0.9 & 0.9 & 0.9 & 0.9 & 1.1 & 1.0 & 1.1 & 0.9 & 1.0 \\
\hline & $\mathrm{s}$ & 0.30 & 0.26 & 0.29 & 0.20 & 0.31 & 0.27 & 0.37 & 0.29 & 0.34 \\
\hline \multirow{2}{*}{ Dry matter } & $\overline{\mathrm{X}}$ & 22.9 & 23.1 & $\begin{array}{r}\text { Ba } \\
23.0\end{array}$ & 22.2 & $23.1^{* *}$ & $\begin{array}{r}\text { Bh } \\
22.7^{-1}\end{array}$ & $24.5^{*}$ & 24.0 & $24.3^{\mathrm{B}}$ \\
\hline & $\mathrm{s}$ & 0.66 & 0.45 & 0.56 & 0.52 & 0.45 & 0.66 & 0.67 & 0.85 & 0.79 \\
\hline \multirow[t]{2}{*}{ Ash } & $\overline{\mathrm{X}}$ & 1.2 & 1.2 & 1.2 & 1.2 & 1.2 & 1.2 & 1.3 & 1.3 & 1.3 \\
\hline & $\mathrm{S}$ & 0.06 & 0.03 & 0.06 & 0.05 & 0.04 & 0.04 & 0.06 & 0.07 & 0.07 \\
\hline \multirow{3}{*}{$\begin{array}{l}\text { Water holding } \\
\text { capacity }\left(\mathrm{cm}^{2}\right)\end{array}$} & & & & Aa & & & $\mathrm{Ah}$ & & & Аа \\
\hline & $\overline{\mathrm{X}}$ & 2.6 & 2.7 & 2.6 & 2.4 & 2.2 & 2.3 & 2.7 & 2.9 & 2.8 \\
\hline & s & 0.53 & 0.62 & 0.57 & 0.55 & 0.33 & 0.46 & 0.54 & 0.53 & 0.054 \\
\hline \multirow[t]{2}{*}{$\mathrm{pH}$} & $\overline{\mathrm{X}}$ & 5.8 & 5.7 & $5.7^{\mathrm{h}}$ & 5.8 & 5.8 & $5.8^{\mathrm{a}}$ & 5.8 & 5.8 & 5.8 \\
\hline & $\mathrm{s}$ & 0.07 & 0.05 & 0.07 & 0.06 & 0.07 & 0.07 & 0.10 & 0.10 & 0.11 \\
\hline \multirow{2}{*}{$\begin{array}{l}\text { Color brightness } \\
\text { (\%) }\end{array}$} & $\overline{\mathrm{X}}$ & $15.6^{* *}$ & 11.7 & $\begin{array}{r}{ }^{\mathrm{B}} \\
13.6\end{array}$ & 13.6 & 13.8 & $\begin{array}{r}\text { B } \\
13.7\end{array}$ & 15.3 & 15.7 & $15.5^{\mathrm{A}}$ \\
\hline & $\mathrm{s}$ & 3.10 & 1.59 & 3.12 & 2.35 & 2.19 & 2.22 & 2.98 & 2.85 & 2.93 \\
\hline
\end{tabular}

Means followed by different letters ( genetics groups) or * (sex) are significantly different:

capital letters or ** - at $\alpha=0.01$; small letters or * - at $\alpha=0.05$

Table 5

Sensory properties (pts) of duck breast muscles (Sensorische Eigenschaften (pkt) der Brustmuskel)

\begin{tabular}{|c|c|c|c|c|c|c|c|c|c|c|}
\hline \multirow{3}{*}{ Specification } & \multirow{3}{*}{$\begin{array}{l}\text { Statis- } \\
\text { tics }\end{array}$} & \multicolumn{9}{|c|}{ Ducks and sex } \\
\hline & & \multicolumn{3}{|c|}{ Muscovy } & \multicolumn{3}{|c|}{ A-44 strain } & \multicolumn{3}{|c|}{ hybrids } \\
\hline & & $\hat{0}$ & q & oto & $\hat{0}$ & $q$ & 19+ & $\hat{0}$ & ㅇ & jot \\
\hline $\begin{array}{l}\text { Aroma: } \\
\text { - intensity }\end{array}$ & $\begin{array}{l}\bar{X} \\
S\end{array}$ & $\begin{array}{c}4.6 \\
0.45\end{array}$ & $\begin{array}{c}4.6 \\
0.42\end{array}$ & $\begin{array}{c}4.6 \\
0.43\end{array}$ & $\begin{array}{c}4.7 \\
0.26\end{array}$ & $\begin{array}{c}4.8 \\
0.25\end{array}$ & $\begin{array}{c}4.8 \\
0.25\end{array}$ & $\begin{array}{c}4.6 \\
0.33\end{array}$ & $\begin{array}{c}4.6 \\
0.46\end{array}$ & $\begin{array}{c}4.6 \\
0.40\end{array}$ \\
\hline - desirability & $\begin{array}{l}\bar{X} \\
\mathrm{~S}\end{array}$ & $\begin{array}{c}4.6 \\
0.45\end{array}$ & $\begin{array}{c}4.6 \\
0.42\end{array}$ & $\begin{array}{c}4.6 \\
0.42\end{array}$ & $\begin{array}{c}4.7 \\
0.26\end{array}$ & $\begin{array}{c}4.8 \\
0.25\end{array}$ & $\begin{array}{c}4.8 \\
0.25\end{array}$ & $\begin{array}{c}4.4 \\
0.31\end{array}$ & $\begin{array}{c}4.5 \\
0.45\end{array}$ & $\begin{array}{c}4.5 \\
0.39\end{array}$ \\
\hline Tenderness & $\begin{array}{l}\bar{X} \\
S\end{array}$ & $\begin{array}{c}4.4 \\
0.50\end{array}$ & $\begin{array}{c}4.5 \\
0.58\end{array}$ & $\begin{array}{c}4.4^{\mathrm{b}} \\
0.53\end{array}$ & $\begin{array}{c}4.7 \\
0.34\end{array}$ & $\begin{array}{c}4.8 \\
0.32\end{array}$ & $\begin{array}{c}4.8^{\mathrm{a}} \\
0.32\end{array}$ & $\begin{array}{c}4.4 \\
0.60\end{array}$ & $\begin{array}{c}4.3 \\
0.71\end{array}$ & $\begin{array}{c}4.4 \\
0.49\end{array}$ \\
\hline Juiciness & $\begin{array}{c}\bar{X} \\
s\end{array}$ & $\begin{array}{c}4.3 \\
0.55\end{array}$ & $\begin{array}{c}4.6 \\
0.50\end{array}$ & $\begin{array}{c}4.4^{\mathrm{h}} \\
0.46\end{array}$ & $\begin{array}{c}4.7 \\
0.33\end{array}$ & $\begin{array}{c}4.8 \\
0.33\end{array}$ & $\begin{array}{c}4.7^{\text {a }} \\
0.33\end{array}$ & $\begin{array}{c}4.4 \\
0.41\end{array}$ & $\begin{array}{c}4.5 \\
0.50\end{array}$ & $\begin{array}{r}\text { h } \\
4.5 \\
0.45\end{array}$ \\
\hline $\begin{array}{l}\text { Palatability: } \\
\text { - intensity } \\
\text { - desirability }\end{array}$ & $\begin{array}{l}\bar{X} \\
S\end{array}$ & $\begin{array}{c}4.3 \\
0.55 \\
\\
4.4 \\
0.50\end{array}$ & $\begin{array}{c}4.5 \\
0.50 \\
4.5 \\
0.58\end{array}$ & $\begin{array}{c}4.4^{\mathrm{b}} \\
0.54 \\
4.4^{\mathrm{b}} \\
0.53\end{array}$ & $\begin{array}{c}4.8 \\
0.34\end{array}$ & $\begin{array}{c}4.8 \\
0.33\end{array}$ & $\begin{array}{c}4.8^{\mathrm{a}} \\
0.33 \\
4.8^{\mathrm{a}} \\
0.31\end{array}$ & $\begin{array}{c}4.5 \\
0.54\end{array}$ & $\begin{array}{c}4.5 \\
0.47 \\
\\
4.5 \\
0.52\end{array}$ & $\begin{array}{c}4.5^{\mathrm{ab}} \\
0.51 \\
4.5^{\mathrm{ab}} \\
0.52\end{array}$ \\
\hline
\end{tabular}

Means followed by different letters ( genetics groups) or * (sex) are significantly different:

capital letters or ** - at $\alpha=0.01$; small letters or * - at $\alpha=0.05$

The results of the heterosis effect estimation in interspecific crossbreeds are presented in Tables 6 and 7. In most cases, especially in the Mullard females and in an evaluation of both sexes, a significant favorable heterosis effect was observed. Its intensity was lower in the crossbred drakes. It probably resulted from a very good 
slaughter value of the Muscovy drakes, and values of their traits were used, among other, for an evaluation of the heterosis effect in the Mullards.

Table 6

Effect of heterosis (\%) on some traits of crossbred ducks (Heterosiseffekt (\%) von Schlachteigenschaften der Enten-Kreuzungen)

\begin{tabular}{|c|c|c|c|}
\hline \multirow[b]{2}{*}{ Specification } & \multicolumn{3}{|c|}{ Sex } \\
\hline & $\hat{0}$ & q & jo오 \\
\hline $\begin{array}{l}\text { Weight of (g): } \\
\text { - body } \\
\text { - carcass } \\
\text { - giblets } \\
\text { - abdominal fat } \\
\text { - edible parts }\end{array}$ & $\begin{array}{c}-15.54 \\
-18.1 \\
-\quad 1.44 \\
-92.44 \\
-18.62 \\
\end{array}$ & $\begin{array}{c}9.94 \\
9.92 \\
25.13 \\
-81.03 \\
6.28 \\
\end{array}$ & $\begin{array}{c}-5.18 \\
-6.71 \\
9.53 \\
-87.73 \\
-8.37 \\
\end{array}$ \\
\hline $\begin{array}{c}\text { Carcass dressing percentage: } \\
\text { I } \\
\text { II }\end{array}$ & $\begin{array}{c}0.09 \\
-5.19\end{array}$ & $\begin{array}{c}2.74 \\
-3.39\end{array}$ & $\begin{array}{c}1.41 \\
-4.28\end{array}$ \\
\hline $\begin{array}{l}\text { Weight in carcass of (g): } \\
\text { - breast muscles } \\
\text { - leg muscles } \\
\text { - total meat } \\
\text { - bones } \\
\text { - skin with fat } \\
\text { - intermuscular fat }\end{array}$ & $\begin{array}{l}-10.05 \\
-19.36 \\
-2.50 \\
-12.00 \\
-42.87 \\
-65.35 \\
\end{array}$ & $\begin{array}{c}39.07 \\
9.12 \\
28.67 \\
29.77 \\
-24.55 \\
-63.10 \\
\end{array}$ & $\begin{array}{c}9.22 \\
-7.66 \\
10.10 \\
4.56 \\
-35.16 \\
-64.35 \\
\end{array}$ \\
\hline $\begin{array}{l}\text { Ratios: } \\
\text { - meat to skin with fat } \\
\text { - meat to bones }\end{array}$ & $\begin{array}{l}75.31 \\
12.93\end{array}$ & $\begin{array}{r}70.87 \\
-2.85 \\
\end{array}$ & $\begin{array}{c}72.87 \\
5.28\end{array}$ \\
\hline $\begin{array}{l}\text { Percentage in carcass of: } \\
\text { - breast muscles } \\
\text { - leg muscles } \\
\text { - remaining muscles } \\
\text { - total meat } \\
\text { - bones } \\
\text { - skin with fat }\end{array}$ & $\begin{array}{c}12.76 \\
0.34 \\
44.42 \\
21.05 \\
4.65 \\
-32.01\end{array}$ & $\begin{array}{c}25.97 \\
-2.37 \\
23.01 \\
16.73 \\
18.58 \\
-32.22\end{array}$ & $\begin{array}{r}18.39 \\
-0.08 \\
33.99 \\
18.60 \\
10.85 \\
-32.00\end{array}$ \\
\hline
\end{tabular}

Table 7

Effect of heterosis (\%) on some meat quality traits of crossbred ducks (Heterosiseffekt (\%) von Fleischqualitätsmerkmalen der Enten-Kreuzungen)

\begin{tabular}{|c|c|c|c|}
\hline \multirow[b]{2}{*}{ Specification } & \multicolumn{3}{|c|}{ Sex } \\
\hline & $\hat{0}$ & q & 19t \\
\hline $\begin{array}{l}\text { Percentage in meat of (\%): } \\
\text { - crude protein } \\
\text { - fat } \\
\text { - dry matter } \\
\text { - ash }\end{array}$ & $\begin{array}{c}1.30 \\
22.22 \\
8.65 \\
8.33\end{array}$ & $\begin{array}{c}1.56 \\
-10.00 \\
3.90 \\
8.33\end{array}$ & $\begin{array}{l}1.30 \\
5.26 \\
6.35 \\
8.33\end{array}$ \\
\hline Water holding capacity (\%) & 8.65 & 20.00 & 14.11 \\
\hline $\mathrm{PH}$ & 0.87 & 1.21 & 1.04 \\
\hline Color brightness (\%) & 4.79 & 23.14 & 13.55 \\
\hline $\begin{array}{l}\text { Aroma: } \\
\text { - intensity } \\
\text { - desirability } \\
\end{array}$ & $\begin{array}{l}-1.08 \\
-5.38 \\
\end{array}$ & $\begin{array}{l}-2.13 \\
-4.26 \\
\end{array}$ & $\begin{array}{l}-2.13 \\
-4.26 \\
\end{array}$ \\
\hline Tenderness & -3.30 & -7.56 & -4.35 \\
\hline Juiciness & -2.22 & -4.26 & -1.10 \\
\hline $\begin{array}{l}\text { Palatability: } \\
\text { - intensity } \\
\text { - desirability }\end{array}$ & $\begin{array}{l}-1.10 \\
-3.23\end{array}$ & $\begin{array}{l}-3.23 \\
-3.23\end{array}$ & $\begin{array}{l}-2.17 \\
-3.23\end{array}$ \\
\hline
\end{tabular}

Positive heterosis in carcasses of the Mullard drakes was noted firs of all in the traits connected with undesirable fatness: He $\widehat{\sigma}$ was equal to $-92.4 \%$ for abdominal fat 
weight, $-42.9 \%$ for the weight of skin with subcutaneous fat, and $-32.0 \%$ for the percentage of skin with subcutaneous fat in a carcass (Table 5). Positive heterosis was also observed in the percentages of breast muscles $(\mathrm{He}=12.8 \%)$ and total meat $(\mathrm{He}>$ $21 \%$ ) in a carcass. These values contributed to a significant heterosis effect in carcasses of drakes as regards the meat to fat with skin ratio $(\mathrm{He}=75.3 \%)$ and the meat to bones ratio ( $\mathrm{He}=12.9 \%$ ). In the Mullard females no positive heterosis was found only in such traits as carcass dressing percentage II ( $\mathrm{He}=-3.6 \%)$, weight of bones in a carcass $(\mathrm{He}=29.8 \%)$, and the percentage of leg muscles and bones in a carcass. Positive heterosis was observed in all other traits of the crossbred ducks, as compared with the Muscovy and A-44 strain females.

An evaluation of the heterosis effect in both sex groups shows that it was present first of all in such traits as the weight and percentage of breast muscles in a carcass (He $9.2 \%$ and $18.4 \%$ respectively), and total meat content of a carcass ( $\mathrm{He}=10.1 \%$ and $18.6 \%$, respectively), as well as in the traits connected with carcass fatness (Table 6).

The heterosis effect estimated for the meat quality traits analyzed are presented in table 7. Its low, negative values for sensory properties indicate slightly worse quality of the meat from the Mullards, compared with the meat from the Pekin ducks and - to a certain extent - with the meat from the Muscovy ducks. Attention should also be paid a considerable, unfavorable value of the He index (He $>8.6 \%$ ) for the water holding capacity of Mullard muscles.

\section{Discussion}

Muscovy ducks, reared in many European countries, are usually characterized by lower fatness, higher meatiness and meat of better quality than Pekin ducks (BAEZA et al., 1998; MULDER, FLETCHER, 1998; PIKUL et al., 1987; PINGEL, 1997; RICARD et al., 1988; ZEIDLER, 1998). According to BOISDRON and GRIMAUD (1991), the breeding work carried out on Muscovy ducks in France since 1968 allowed to increase body weights of drakes and ducks by $80 \mathrm{~g}$ and $40 \mathrm{~g}$ respectively each year. Since 1990 their body gains were $50 \mathrm{~g}$ and $30 \mathrm{~g}$ respectively. Therefore, Muscovy drakes of heavy strains may reach body weights of $5100 \mathrm{~g}$ in the $12^{\text {th }}$ week of rearing, and Muscovy ducks $-2800 \mathrm{~g}$ in the $10^{\text {th }}$ week of rearing. The results concerning body weights of Muscovy ducks obtained in the present experiment are much lower than those reported by BOISDRON and GRIMAUD (1991), but generally do not differ much from those presented by other authors (BAEZA et al., 1998; GÓRSKI et al., 1999; GUY et al., 1995; LEWCZUK et al.,1992; PIKUL et al., 1987; PINGEL, TRETTNER, 1987; RICARD et al., 1988).

Pekin ducks are characterized by a high growth rate and a considerable meat content of a carcass (KSIAZŻKIEWICZ, 1995; LEWCZUK et al., 1992). Their commonly-known defects are excessive fatness and a low percentage of breast muscles in a carcass (SZASZ and BOGENFÜRST, 1998). The body weights of A-44 ducks achieved in own investigations ( $\overline{\mathrm{x}}$ ca. $2870 \mathrm{~g}$ ) were considerably higher than reported in previous studies (LEWCZUK et al., 1992). In the studies conducted by ISGUZAR et al. (2002) on the Turkish population of Pekin ducks their body weights at the age of eight weeks were as low as $1.7 \mathrm{~kg}$ in females and $1.9 \mathrm{~kg}$ in males. However, according to PINGEL Pekin ducks of commercial strains with improved meatiness may be characterized by high body weights at seven to eight weeks of age, i.e. $2.6-3.0 \mathrm{~kg}$ and $3.6-4.0 \mathrm{~kg}$ for females and males respectively. High body weights $(\bar{x}>3.3 \mathrm{~kg})$ of seven-week-old 
Pekin ducks reared according to different breeding programmes were also reported by FARHAT and CHAVEZ (2000).

The average body weights of crossbreeds (Muscovy $\lesssim$ x Pekin $\bigcirc$ ) may vary from 2600 $\mathrm{g}$ to $3900 \mathrm{~g}$ (BABILE et al., 1988; MAZANOWSKI, BERNACKI, 2000; RETAILLEAU, 1997; WAWRO et al., 2001; WILKIEWICZ-WAWRO et al., 1994) or even over $4000 \mathrm{~g}$ (BAEZA et al., 2000), depending on sex and age at slaughter (1012 weeks). This indicates that the experimental crossbred ducks aged 12 weeks were characterized by mean body weights.

The differences in carcass meatiness between the Muscovy ducks and hybrids, noted in the present experiment and resulting from sexual dimorphism in body weights, are consistent with the observations by RICARD, (1986). GILLE and SALOMON (1998), who compared the growth in breast and leg muscles in ducks, report that the total weight of these muscles in males and females aged 80 days was the lowest in Muscovy ducks, higher - in Pekin ducks, and the highest - in their crossbreeds. This results probably from a lower growth rate of Muscovy ducks in the initial period of rearing, as compared with Pekin ducks.

Great demand for ducks characterized by low or average body weights, high meatiness and low fatness (WAWRO et al., 2001), observed in many countries for ducks with low or average body weight, well-developed musculature and low fatness (WAWRO et al., 2001), may be satisfied by increased production of interspecific hybrids (Mullards), which seem to meet the above requirements. In their carcasses the meat to skin with fat ratio was much better than in carcasses of the Muscovy and A-44 ducks. Very good meat to fat and meat to bones rations in Mullard carcasses were also stressed by WILKIEWICZ-WAWRO (1994) and WAWRO et al. (2001).

The results of own studies concerning the percentages of particular tissue components in duck carcasses confirm those obtained by other authors. WILKIEWICZ-WAWRO (1994) reports that in Mullard carcasses the meat content is over 54\%, and the skin with fat content - below 22\%. A comparative analysis of Muscovy ducks, Pekin ducks and their hybrids (Muscovy male x Pekin female) made by GÓRSKI et al. (1999) shows that carcasses of crossbreeds were characterized by the lowest fatness. According to SZASZ and BOGENFÜRST (1998), carcass fatness of Muscovy ducks and hybrids is at a similar level, much lower compared with Pekin ducks. Also BOISDRON, GRIMAUD (1991), and LEWCZUK et al. (1992) emphasize lower fatness of Muscovy ducks compared with Pekin ones.

The nutritive and dietary value, palatability and technological suitability of poultry meat depend not only on the species and age of fowls, but also on the correlations between chemical components, and the physicochemical properties of muscles.

The results obtained do not provide the basis for stating that the meat from Mullards is characterized by better chemical composition than the meat from Muscovy and Pekin ducks.

The concentrations of particular chemical components in the meat from the experimental ducks were generally similar to those reported by SOBINA et al. (1987) for ducks of the A-44 strain, and by WILKIEWICZ-WAWRO (1994) for Mullards. BAEZA et al., (2000) point to age-changes in the levels of chemical components in breast muscles of Mullards. Muscles of older ducks (aged 12 weeks) contain significantly more protein, lipids and mineral components, and less water, compared with those of younger ones (aged eight to ten weeks). A decrease in $\mathrm{pH}$ of breast 
muscles, accompanied by higher juice losses can be observed in Muscovy males and females as they grow older (BAEZA et al., 1998). A lighter color of meat from Muscove ducks, compared with drakes, results from higher and faster fat deposition as well as earlier maturation of females.

The differences noted in sensory meat quality may result from different age of ducks at slaughter, but first of all from a high intermuscular fat content of Pekin duck muscles. A significant effect of age on meat quality in Pekin ducks was previously reported by SOBINA et al. (1987). Muscles of ducks slaughter at seven to eight weeks of age show the highest nutritive value and culinary quality. According to BAEZA et al. (1998), the quality and palatability of breast muscles of Muscovy ducks deteriorate with age. They become less juicy and more tough (less tender), which is connected with decreased collagen solubility accompanied by increased thickness of muscle fibres.

Heterosis is a genotypic result of specific combining ability of crossbred strains of fowls. It is usually presented as the domination of the average performance of the first generation-hybrids over the average performance of their parents. Heterosis origins from non-additive genetic effects (domination, overdomination, epistasis). Higher values of the heterosis effect should be expected in interspecific hybrids than interstrain ones. The fact that available literature provides no information on the heterosis effect in relation to slaughter value traits in Mullards makes discussion on this topic difficult. However, the results obtained allow to state that a favorable heterosis effect can be expected in interspecific hybrids, positive in carcass meatiness and negative in carcass fatness.

The heterosis effects estimated for meat quality traits turned out to be unequivocal. The He values at a level of $\pm 1 \%$ should be considered insignificant. The negative values of the He index indicate not too high sensory quality of the meat from Mullards. The high value of the heterosis index ( $\mathrm{He}>8.6 \%)$ for the water holding capacity of muscles shows worse quality of the meat from crossbreeds, compared with the meat from Muscovy and Pekin ducks. Considerable drip of free water in the meat from Mullards indicates its limited suitability for processing. The above data allow to make a general statement that no favorable heterosis effects can be found in most meat quality traits in Mullards.

\section{References}

BABILE, R.; AUVERGENE, A.; DELPECH, P.; CLAVIER, E.:

Influence d'un retionnement precoce lez performances de decoupe de types genetiques de canards de Barbarie. "La genetique de canard de Barbarie (Carina moschata) et du mulard". Toulouse-Auzeville (France). Colloques de I' INRA, 42 (1988), 101-109

BAÉZA, E.; SALICHON, M.R.; MARCHE, G.; JUIN, H.:

Effect of sex on growth, technological and organoleptic characteristics of Muscovy duck breast muscle.Brit. Poultry Sci., 39 (1998), 3, 398-403

BAÉZA, E.; SALICHON, M.R.; MARCHE, G.; WACRENIER, N.; DOMINGUEZ, B.; CULIOLI, J.:

Effect of age and sex on the structural, chemical, technological characteristics of mule duck meat. Brit. Poultry Sci., 41 (2000), 3, 300-307

BARYŁKO-PIKIELNA, N.; KOSSAKOWSKA, T.; BALDWIN, Z.:

The selection of on optimal method to prepare beef and pork for the sensoric evaluation. Roczniki Instytutu Przemysłu Mięsnego, 1 (1964), 132-139

BOISDRON, P.; GRIMAUD, R.:

The Muscovy duck: a quality product with a bright future. Misset World Poultry, 7 (1991), 7, 12-13 
FARHAT, A.; CHAVEZ, E.R.:

Comparative performance of blood chemistry and carcass composition of two lines of Pekin ducks reared mixed or separated by sex. Poultry Sci., 79 (2000), 460-465

GILLE, U.; SALOMON, F-V.: Muscle growth in wild and domestic duck. Brit.Poultry Sci., 39 (1998), 4, 500-505

GÓRSKI, J.; WITAK, B.; PIETKIEWICZ, M.:

Comparison between body weight and feed consumption in meat ducks, Muscovy ducks and their crossbreds during rearing. Rocz. Nauk. Zoot. 26 (1999), 3, 111-122

GUY, G.; ROUSSELOT-PAILLEY, D.; ROUVIER, R.:

Description de la croissance de canettes mulardes. Ann. Zootech., 47 (1998), 4, 303-310

HOFFMAN, E.:

A history of The Domestic Muscovy Duck. In: $10^{\text {th }}$ World's Poultry Sci. Waterfowl Symp., Halle, Germany (1995), 477-523

ISGUZAR, E.; KOCAK, C.; PINGEL, H.:

Growth, carcass traits and meat quality of different local ducks and Turkish Pekings. Arch. Tierz., Dummerstorf 45 (2002), 4, 413-418

KSIĄŻKIEWICZ, J.M.:

Duck breeding strategy. In: $11^{\text {th }}$ Proc. Inter. Symp. "Current Problems in Avian Genetics", Balice (1995), 111-119

LEWCZUK, A.; BOCHNO, R.; WAWRO, E.:

Slaughter value of muscovy and Pekin of A-44 strain ducks. Prace i Materiały Zoot., 42 (1992), 83-90

MAZANOWSKI, A.; BERNACKI, Z.:

Rearing and fattening performance of variously fed crossbreds of muscovy drakes and Pekin- type ducks. Ann. Anim. Sci., Rocz. Nauk. Zoot., 27 (2000), 4, 181-199

MULDER, R.W.A.W.; FLETCHER, D.L.:

Poultry meat science towards the 21 st century. Proc. $10^{\text {th }}$ European Poultry Conf., Jerusalem, Israel, 1 (1998), 121-125

PIKUL, J.; DORUCHOWSKI, W.; TAŃSKI, S.; REKSIŃSKI, T.:

Slaughter yields, carcass composition, chemical analysis and technological properties of muscovy and Pekin ducks. Zesz. Nauk. Drobiarstwo, COBRD Poznań, 4 (1987), 73-92

PINGEL, H.; TRETTNER, K.:

Mast- und Schlachtleistung von Moschusenten (Carina moschata) und deren Kreuzungen mit Pekingenten (Anas plathyrinchos). Arch. Tierz., Berlin 30 (1987), 523-527

PINGEL, H.:

Perspectives of the production of waterfowl. In: $11^{\text {th }}$ European Symp. on Waterfowl, Nantes, France, (1997), 128-134

POHJA, M.S.; NINIVAARA, F.P.:

Die Bestimmung der Wasserbedinung des Fleisches mittees der Konstant-druck-methode. Fleischwirtschaft, 9 (1957), 193-195

RETAILLEAU, B.:

Performances of roasting mule duck testing of various genotypes and of various slaughter ages. Proc. 11 th European Symp. on Waterfowl, Nantes, France (1997), 633-638

RICARD, F.H.:

Composition anatomique de la carcasse du canard mulard comparée aux deux types parentaux. Conference Avicole, Paris, WPSA, 3 (1986), 47-64

RICARD, F.H.; de CARVILLE, H.; MARCHE, G.:

Etude comparative de la composition anatomique des canards de Barbarie, Pekin et Mulard. "La genetique de canard de Barbarie (Carina moschata) et du mulard". Toulouse-Auzeville (France). Colloques de I'INRA, 42 (1988), 74-97

SOBINA, I.; BOCHNO, R.; MELLER, Z.; KONDRATOWICZ, J.:

Comparison of certain physicochemical parameters of raw meat from ducks killed at different age. Acta Acad. Agricult. Techn. Olst., Zootechnica 30 (1987), 185-192

SZÁSZ, S.; BOGENFÜRST, F.:

Study on body fat content in Pekin, Muscovy and mule ducks. Proc. 10 th Europ. Poultry Conf., Jerusalem, Israel, 2 (1998), 719-722

WAWRO, K.; BOCHNO, R.; WILKIEWICZ-WAWRO, E.:

Slaughter value of crossbred ducks (Muscovy $\times$ Pekin) slaughtered at a different age. Natur. Sc., 8 (2001), 17-25

WAWRO, K.; WILKIEWICZ-WAWRO, E.; PRUSINOWSKA, I.; KONCICKI, A.; KRASNODĘBSKADEPTA, A.:

The selected blood indices of Muscovy and Pekin ducks and their crossbreds and evaluation of heterosis effect. Zesz. Nauk. PTZ 49 (2000), 177-190 
WILKIEWICZ-WAWRO, E.:

Effects of rearing and slaughter value of interspecific hybrid ducks fed ad libitum or a limited ration. Acta Acad. Agricult. Techn. Olst., Zootechnica, 40, Supplementum B (1994), 3-37

ZEIDLER, G.:

Poultry products in the 21 st century. In: Proc. 10 th Europ. Poultry Conf., Jerusalem, Israel 1 (1998), $132-141$

Received: 2003-04-14

Accepted: 2004-05-11

Authors' address

Prof. Dr. habil. KAZIMIERZ WAWRO, Dr. habil. ELŻBIETA WILKIEWICZWAWRO, Mgr. KATARZYNA KLECZEK, Dr. WIESŁAW BRZOZOWSKI

Department of Breeding Methods and Animal Improvement, University of Warmia and Mazury in Olsztyn, Poland

ul. M. Oczapowskiego 5

10-718 Olsztyn

Poland 